Background. This survey was conducted as part of routine service provision analysis by the psychotherapy department. It aimed to assess the level of satisfaction patients feel towards their experience of attending for psychotherapy, in order to inform local management on the service being offered by the department. Ethics committee confirmed this fulfilled "Service evaluation" criterion and the project was registered with the local NHS quality improvement register.

Method. Patients who completed an episode of therapy were invited to complete a survey form. This consisted of a Client Satisfaction Questionnaire (CSQ-8) as well as four additional questions pertaining to patient satisfaction. The patient's therapist would inform administration staff of the patient's final appointment; administration staff would then issue the patient with a questionnaire which they were invited to complete and return in their own time. The questionnaires were completed anonymously and no reward was offered for completing the questionnaire. The therapies included group analysis, psychodynamic individual and Cognitive Analytic Therapy.

Result. 22 patients who had completed psychotherapy in 20182019 returned a completed questionnaire. The average and range responses were examined.

The average response was " 4 : Excellent" for the overall rating of the service received, and for 5 other questions on the CSQ- 8 the average score was the highest possible. The average response was slightly lower on the question about whether the service met their needs "3: Mostly", and on the question: Has the service you received helped you to deal more effectively with your problems? (3 yes, somewhat). The additional questions highlighted how important the setting and administration role played in the experience of therapy. The questionnaire also included a free text box giving the patient the opportunity to offer any other comments. Many of these included messages of gratitude and remarks on the impact therapy has had on their general wellbeing.

Conclusion. In general it is encouraging to see that feedback provided through this survey was extremely positive. This was reflected both in the Likert scale questions and the free text box. Patients are described themselves as very satisfied with their experience within therapy and reflected a positive experience of the holding environment provided by the department as a whole.

(NO Funding received)

\section{The role of telephone consultations in psychiatry}

Jonathan Packer ${ }^{1 \star}$, Emma Fisher ${ }^{2}$, Anne-Marie Simons², Kirsty Bolochowecki², Benita Roff ${ }^{2}$, Sanjay Khurmi ${ }^{2}$ and Luke Jones ${ }^{3}$

${ }^{1}$ Herefordshire and Worcestershire Health and Care Trust; ${ }^{2}$ Coventry and Warwickshire Partnership Trust and ${ }^{3}$ Warwick Medical School ${ }^{*}$ Corresponding author.

doi: 10.1192/bjo.2021.889

Aims. Telephone consultations have been in clinical use since the early 1960s and are increasing in frequency and importance in many areas of medicine. With the advent of the COVID-19 pandemic in 2020, the use of telemedicine consultations increased dramatically alongside utilization of other digital technologies. Despite promise and potential advantages for clinicians (including remote working, improved time management and safety) there are known drawbacks to telephone consultations for psychiatrists. This includes limitations to assessments of mental state and risk, with loss of non-verbal communication often cited as a point in favour of more sophisticated technologies such as video calling. By adopting telephone consultations to a greater extent during the initial months of the COVID-19 pandemic in the Coventry Crisis Resolution and Home Treatment Team (CRHTT), we aimed to assess the patient experience in telehealth, through a patient survey.

Method. After an initial assessment or follow-up consultation with a medical practitioner from the crisis team, patients were invited to take part in a short questionnaire with a member of the administration staff. This consisted of eight questions on a Likert scale and three open questions for comments. Results were collated and analyzed via Microsoft Excel.

Result. Most patients found the telephone consultations satisfactory, with more than $90 \%$ returning positive scores in understanding, convenience and overall satisfaction. All patients felt listened to and that their confidentiality was maintained; with all but one respondent willing to engage in further consultations via the telephone. Negative scores were typically returned for practical telephonic problems including poor signal, interference and background noise. In their comments patients expressed largely positive views about their experience with their clinician; analysis revealed key insights into the patient experience, demonstrating the convenience, comfort and flexibility possible with 'telepsychiatry'.

Conclusion. Patient experience of telemedicine in a UK psychiatric crisis team is mostly positive, with clear advantages for both patients and clinicians. Our results show telephone consultations can be expanded to new patient assessments alongside follow-ups, enabling the team to reach a greater number of service users. This includes service users who are housebound due to infirmity, required to shield or have significant anxiety about the pandemic.

\section{Profile, referral pathways and re-attendance of psychiatric patients attending the emergency department: focus on suicidality \& self-harm}

\author{
Haridha Pandian ${ }^{1 \star}$ and Nilamadhab $\operatorname{Kar}^{2}$ \\ ${ }^{1}$ Royal Wolverhampton NHS Foundation Trust and ${ }^{2}$ Black Country \\ Healthcare NHS Foundation Trust \\ ${ }^{\star}$ Corresponding author.
}

doi: 10.1192/bjo.2021.890

Aims. The number of patients presenting to Emergency Departments (EDs) in the UK with acute psychiatric issues is a major concern. This project aimed to explore the outcome of patients assessed by Mental Health Liaison Services (MHLS) in a large district general hospital ED in the UK, with a focus on patients with self-harm or suicidality.

Method. Data were extracted from electronic patient records on 100 consecutive attendees to MHLS in July 2020. Data were collected on demographics, index of multiple deprivation (IMD) by postcode, time and reason for attendance, known ICD-10 diagnoses, self-harm history, alcohol/substance misuse at time of presentation, recent psychosocial stressors and outcome of MHLS assessment. Assessments by MHLS in the preceding 12 months and reattendance to the service within 3 months following this assessment were also recorded.

Result. The sample included 44 male and 56 female patients, with a mean age of 35.3 years. $80.0 \%$ of patients were Caucasian. $67.0 \%$ lived in areas classed within the top $30 \%$ most deprived in the country, whilst $2.0 \%$ had no fixed abode. The majority $(79.0 \%)$ of patients self-presented; outside of normal working hours (70.0\%). The most common reasons for attendance were thoughts/intent of self-harm/suicide (50.0\%), overdose $(29.0 \%)$ and self-harm by laceration (7.0\%). 
The majority (73.0\%) of patients had a known psychiatric diagnosis, with the most frequent being depressive disorder (36.0\%) and emotionally unstable personality disorder (15.0\%). Almost half (48.0\%) had a history of self-harm, and $40.0 \%$ were under the influence of alcohol/illicit substances upon presentation to ED. The most common psychosocial triggers were conflict with partner (26.0\%) and stress due to the COVID-19 pandemic (19.0\%).

Following assessment, $24.0 \%$ of patients were discharged to their General Practitioner, $18.0 \%$ to the community mental health team; and $17.0 \%$ to the Crisis \& Home Treatment Team. A minority (13.0\%) were admitted to a psychiatric hospital (10.0\% informally, 3.0\% under the Mental Health Act 1983).

Approximately one in five (21.0\%) patients re-attended to MHLS within 3 months. Out of 37 patients that had previously been assessed by MHLS in the preceding 12 months; $37.8 \%$ were reassessed within 3 months $(\mathrm{p}<0.01)$.

Conclusion. In the studied sample, most (90\%) of psychiatric patients attended ED for self-harm or suicide, and a significant proportion had repeat attendance. Socioeconomic deprivation, substance misuse, relationship difficulties and stress due to the COVID-19 pandemic were major issues, alongside diagnosed depression and personality disorder. Focussed support for these issues may decrease ED attendance due to self-harm/suicidality.

\section{A description of the use of zuclopenthixol decanoate long-acting injection in a large mental health trust \\ Shay-Anne Pantall ${ }^{1 *}$, Emily Whitehouse ${ }^{2}$ and Lisa Brownell ${ }^{1}$ \\ ${ }^{1}$ Birmingham and Solihull Mental Health NHS Foundation Trust and ${ }^{2}$ University of Birmingham, Medical School \\ ${ }^{\star}$ Corresponding author.}

doi: 10.1192/bjo.2021.891

Aims. Adherence with antipsychotic medication is an important factor in the prevention of relapse in psychotic disorders such as schizophrenia. Long acting antipsychotic injections promote improved adherence. In recent years, second generation antipsychotic long-acting injections have become increasingly popular, and little has been written about the use of the older depot medications. Here, we explore the current use of one of the firstgeneration antipsychotic long acting injections in Birmingham and Solihull Mental Health NHS Foundation Trust.

Method. An 18-month retrospective case-note review of all patients who started zuclopenthixol decanoate during the first 6 months of $2018(n=45)$

Result. Key findings included: -

\section{- $71 \%$ were male}

- The mean age was 37 (range 19-65)

- The most common diagnoses were: schizophrenia (51\%), bipolar affective disorder (18\%) and schizoaffective disorder (13\%). We noted that 2 individuals $(4 \%)$ had a primary diagnosis of recurrent depressive disorder, $2(4 \%)$ had a primary diagnosis of emotionally unstable personality disorder.

- $60 \%$ of those who were prescribed zuclopenthixol decanoate discontinued it within the 18-month follow-up period.

- The vast majority of discontinuation occurred within the first 6 months, and after this, few individuals stopped treatment.

- The most common reason for discontinuation was side effects (57\%), with other reasons including patient choice (7\%), noncompliance $(7 \%)$, pregnancy $(4 \%)$, or needle phobia $(4 \%)$.

Conclusion. Zuclopenthixol decanoate has been used for individuals with both schizophrenia and paranoid psychosis (where it is licenced) and also occasionally for other indications. A high proportion discontinued the zuclopenthixol within 6 months, this generally being attributed to adverse effects. Those who were still receiving this medication at 6 months were very likely to continue to take it throughout the 18 months. We would therefore recommend robust monitoring for and management of adverse effects in the early phases of treatment.

The clinical complexity of cases of schizophrenia in a community mental health team: a 3 year comparison

Shay-Anne Pantall ${ }^{1 \star}$, Laxsan Karunanithy ${ }^{2}$, Hayley Boden ${ }^{2}$ and Lisa Brownell ${ }^{1}$

${ }^{1}$ Birmingham and Solihull Mental Health NHS Foundation Trust and ${ }^{2}$ University of Birmingham, Medical School

${ }^{*}$ Corresponding author.

doi: 10.1192/bjo.2021.892

Aims. To describe the changes in complexity and management of individuals with schizophrenia in a community mental health team (CMHT) over a three year period.

Background. It is often believed that individuals receiving care from CMHTs are those with low levels of complexity and risk, and are relatively stable, with more complex individuals being managed by assertive outreach or other specialist teams. Here, we describe changes in the complexity, comorbidity, service-usage and management, of patients with a diagnosis of schizophrenia in a CMHT between 2016 and 2019 .

Method. Data were collected from an electronic patient record system (RiO) for all individuals with schizophrenia in a CMHT in Birmingham ( $\mathrm{n}=84$ in 2016, $\mathrm{n}=71$ in 2019), examining demographic variables, comorbidity, use of mental health services and current management.

Result. Key findings included: -

- $63 \%$ were managed through care programme approach (CPA) in 2016 , compared to only $31 \%$ in 2019 .

- $21 \%$ had required home treatment or inpatient care in the preceding 12 months in 2016; this had improved to 8.5\% in 2019.

- Significant levels of psychiatric comorbidity, including addictions with almost half of patients (46.5\%) having a known history of substance use in 2019 , compared to only $15.5 \%$ noted in 2016.

- Pharmacological management has remained broadly similar; in $201621 \%$ patients were taking a combination of 2 antipsychotics compared to only $10 \%$ in 2019 and $25 \%$ were taking clozapine in 2016 (21\% in 2019). 39\% were prescribed a long acting antipsychotic injection in 2016, compared to $32 \%$ in 2019.

- In 2016, medication was being prescribed in the majority of cases within secondary care (55\%) patients and in primary care in only $21 \%$. GPs have now taken on greater prescribing responsibility in 2019 , prescribing in $44 \%$ of cases, with $47 \%$ being prescribed by the CMHT.

Conclusion. The acuity and management of individuals with a diagnosis of schizophrenia under the care of a CMHT has changed over a 3 year period. It is positive to note the reduced use of crisis services and lower rates of polypharmacy. There is a reduction in the proportion of patients receiving management through $\mathrm{CPA}$, and a move towards more medication being prescribed in primary care. The reasons for this change are however unclear and may reflect change in available resources, given that more than half of this group receive clozapine or long acting injections, and have high levels of comorbidity. 